\title{
Editorial for intelligent interactive multimedia systems and services
}

\author{
Ernesto Damiani • Robert J. Howlett • Lakhmi C. Jain • \\ Jechang Jeong
}

Published online: 20 April 2012

(C) Springer Science+Business Media, LLC 2012

Users worldwide are spending more and more of their time on smart device platforms providing ubiquitous multimedia experience. Smart phones, tablets, e-Readers, web enabled television sets and other device platforms are all participating in this revolution. From the point of view of human-system interaction, however, such platforms pose distinct research problems. A consumer engaged in playing fantasy football has different interaction needs to one researching an address on a cell phone. Today, the vast majority of publishers simply do not have the ability to provide both the scale and quality of rich content to offer a usage experience targeted for multiple platforms. This special issue of MTAP devoted to "Intelligent Interactive Multimedia Systems and Services" is aimed at providing an overview of recent research results in this hot area, highlighting interdisciplinary aspects as well as the links between basic multimedia techniques and smart device applications. We believe that all papers featured in this special issue, while providing outstanding research contributions, succeeded in remaining clear and understandable enough to have a string appeal for nonspecialists wishing to understand the state of the art in this field.

Some papers, like the one by Othmani et al. (DOI 10.1007/s11042-010-0697-6) "A novel approach for high dimension 3D object representation using Multi-Mother Wavelet Network" deal with basic research results in the representation and processing of multimedia objects.

Other papers cover advanced human-system interaction; for instance, the paper by Sakurai et al. "A retrieval method adaptively reducing user's subjective impression gap" (DOI 10.1007/s11042-010-0690-0) deals with improving the user's perception of retrieval

E. Damiani $(\bowtie)$

Università degli Studi di Milano, Milan, Italy

e-mail: ernesto.damiani@unimi.it

R. J. Howlett

University of Brighton, Brighton, UK

L. C. Jain

University of South Australia, Adelaide, Australia

J. Jeong

Hanyang University, Seoul, South Korea 
support, while the paper by Alepis et al. (DOI 10.1007/s11042-011-0744-y) "Multimodal object oriented user interfaces in mobile affective interaction" presents a new vision of interface multimodality. The papers by Ozturk et al. "Real-time tracking of humans and visualization of their future footsteps in public indoor environments" (DOI 10.1007/s11042010-0691-z) and by Anisetti et al. (DOI 10.1007/s11042-010-0721-x), "Landmark-assisted location and tracking in outdoor mobile network" pave the way to multi-party applications based on location tracking. New interaction paradigms are also discussed in the paper by Vatavu et al. (DOI 10.1007/s11042-010-0698-5), "Point \& click mediated interactions for large home entertainment displays".

Another set of papers focuses on the ongoing evolution of smart devices. The papers by Li et al. (DOI 10.1007/s11042-010-0692-y) "A low-cost projector-based hand-held flexible display system", by Jeon et al. (DOI 10.1007/s11042-010-0694-9) “Application for deinterlacing method using edge direction classification and fuzzy inference system" by Nafaa et al. (DOI 10.1007/s11042-011-0755-8) “A dependable multisource streaming system for peer-to-peer -based video on demand services provisioning" and by Chen et al. (DOI 10.1007/s11042-011-0746-9) "Face-based multiple instance analysis for smart electronics billboard" provide valuable insight on features that will be incorporated in the next generation of devices.

A number of interesting papers propose new applications to be supported by smart device platforms.

Some of them focus on personal entertainment. The paper by Lampropoulos et al. "A Cascade-Hybrid Music Recommender System for mobile services based on musical genre classification and personality diagnosis" (DOI 10.1007/s11042-011-0742-0) opens a new research line in the field of recommender systems for music, while new games are discussed in the paper by Guo et al. (DOI 10.1007/s11042-010-0711-z) "Design-in-play: improving the variability of indoor pervasive games". Figueirêdo et al. bridge the gap between multimedia data and semantic annotations in their paper "PhotoGeo: a photo digital library with spatial-temporal support and self-annotation" (DOI 10.1007/s11042-011-0745-x); semantics-aware content adaptation is also discussed in the paper by Rijsselbergen et al. "Semantic Mastering: content adaptation in the creative drama production workflow" (DOI 10.1007/s11042-010-0710-0).

Other applications envision smart devices as a part of large-scale service platforms like the healthcare one described in the paper by Vecchia et al. "An infrastructure for smart hospitals" (DOI 10.1007/s11042-010-0695-8). Finally, two interesting papers deal with device-related aspects of a growing field, Multimedia Security. The paper by Kumar et al.

"OS-Guard: on-site signature based framework for multimedia surveillance data management", (DOI 10.1007/s11042-010-0693-x) and the one by Chung et al. "Intelligent copyright protection system using a matching video retrieval algorithm" (DOI 10.1007/s11042-011-0743-z) shed new light on the time-honored problems of handling surveillance data securely and of protecting intellectual property in video retrieval systems.

Putting together a special issue like this is always a team effort. First of all we would like to thank the anonymous referees who selected these papers and suggested improvements and corrections. Thanks are also due to all the authors who selected MTAP and this special issue as the right venue to present the outcome of their research. 


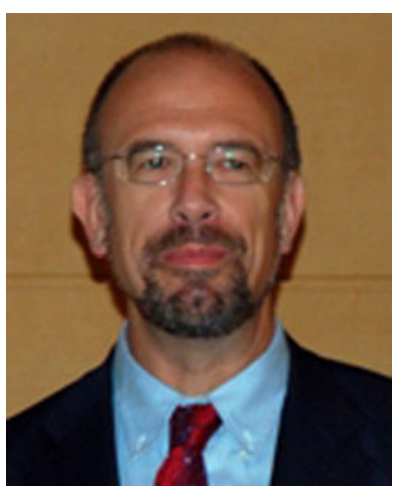

Ernesto Damiani is currently a Professor at the Università degli Studi di Milano and the director of the Università degli Studi di Milano's Ph.D. program in Computer Science. His areas of interest include mobile networks, business process representation, Web services security, processing of semi and unstructured information (e.g., XML), models and platforms supporting open source development, and semantics-aware content engineering for multimedia. He is an Associate Editor of the IEEE Transactions on Service Oriented Computing, Area Editor of the Journal of System Architecture and a member of various editorial boards. He has published several books and about 200 papers and international patents. Prof. Damiani is a Senior Member of the IEEE and ACM Distinguished Scientist.

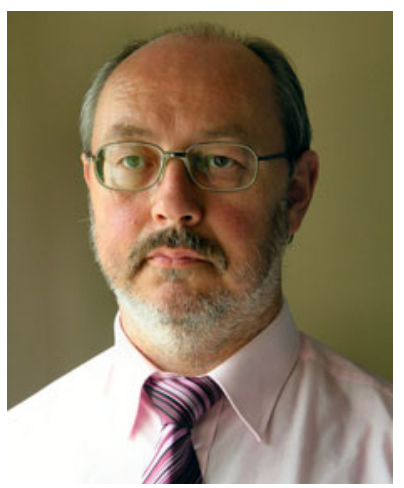

Robert J. Howlett is a member of the Institution of Engineering and Technology and the British Computer Society. He is a Chartered Engineer and a Chartered Information Technology Practitioner.

$\mathrm{He}$ is the Executive Chair of the KES (Knowledge-Based and Intelligent Engineering Systems) International, an organisation dedicated to supporting and facilitating research, in three areas: intelligent systems; sustainability in energy and buildings; and innovation and knowledge transfer.

$\mathrm{He}$ is a Director of the Institute of Knowledge Transfer. He is a nationally known figure in knowledge transfer and the UK Government Knowledge Transfer Partnerships (KTP) programme. His work on knowledge transfer and innovation has been recognised by his appointment as Visiting Professor, Enterprise, at Bournemouth University. He has supervised about 20 projects transferring university expertise to companies, mainly small to medium enterprises (SMEs), and has set up many more.

He has a number of years experience of applying neural-networks, expert systems, fuzzy paradigms and other intelligent techniques to industrial problem domains e.g. sustainability; control, modelling and 
simulation of renewable energy systems; monitoring and control of internal combustion engines, particularly small engines for off road and power generation applications; and a range of condition monitoring and fault diagnosis problems. He led a research team, funded by grants and industrial contracts. He has published widely on the subject, and has presented invited talks, keynote addresses etc.

$\mathrm{He}$ is Editor-in-Chief of the International Journal of Knowledge-Based Intelligent Engineering Systems and Honorary Editor of Intelligent Decision Technologies: an International Journal. He supports a number of other journals through regional editorships and membership of advisory and review boards. He is a past and current member of the scientific committees of a number of conferences. He is on the editorial board of various book series. He has authored over 50 publications in refereed journals and conferences, and edited over 20 books. He has reviewed research project applications for the EPSRC, and internationally as an Expert Evaluator under Framework and for other EU programmes.

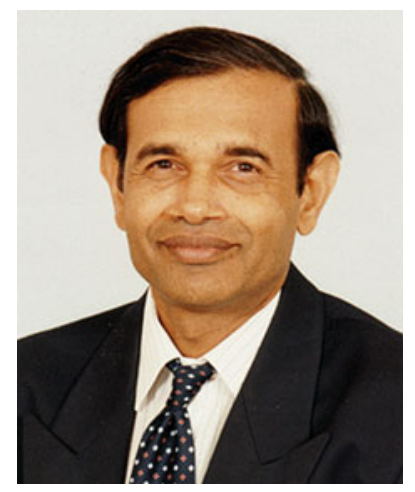

Lakhmi C. Jain Professor Lakhmi Jain, BE(Hons), ME, PhD, is a Director/Founder of the Knowledge-Based Intelligent Engineering Systems Centre. His interests focus on the novel techniques such as knowledge-based intelligent machines, virtual systems, intelligent agents, and the application of these techniques in areas such as engineering, science, aviation, healthcare, defence and so on.

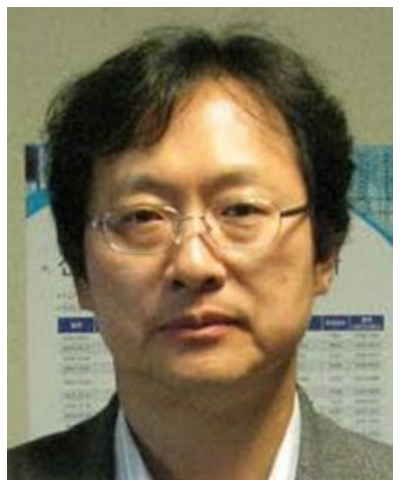

Jechang Jeong received a BS degree in electronic engineering from Seoul National University, Korea, in 1980, an MS degree in electrical engineering from the Korea Advanced Institute of Science and Technology in 1982, and a PhD degree in electrical engineering from the University of Michigan, Ann Arbor, in 1990. From 1982 to 1986, he was with the Korean Broadcasting System, where he helped develop teletext systems. From 1990 to 1991, he worked as a postdoctoral research associate at the University of Michigan, Ann Arbor, where 
he helped to develop various signal-processing algorithms. From 1991 through 1995, he was with the Samsung Electronics Company, Korea, where he was involved in the development of HDTV, digital broadcasting receivers, and other multimedia systems. Since 1995, he has conducted research at Hanyang University, Seoul, Korea. His research interests include digital signal processing, digital communication, and image and audio compression for HDTV and multimedia applications. He has published numerous technical papers. He received the Scientist of the Month award in 1998, from the Ministry of Science and Technology of Korea, and was the recipient of the 2007 IEEE Chester Sall Award and 2008 ETRI Journal Paper Award. He was also honored with a government commendation in 1998 from the Ministry of Information and Communication of Korea. 